\title{
Dinâmicas Sociais e Estados de Humor
}

\author{
Flávio Rebustini \\ Afonso Antonio Machado
}

Laboratório Estudos e Pesquisas em Psicologia do Esporte (LEPESPE),

Departamento de Educação Física, IB/UNESP - Universidade Estadual Paulista, Rio Claro, SP, Brasil

Resumo: O objetivo do estudo foi avaliar as relações das dinâmicas sociais (família, amigos e escola; i.e., três fatores sociais) sobre os estados de humor. A pesquisa foi realizada com uma equipe feminina de voleibol (14/15 anos). Aplicou-se o teste POMS (Profile of Mood States) para mensurar os estados de humor. Foi utilizada uma escala de 1 (muito mal) a 5 (muito bem) para que as atletas avaliassem cada um dos três fatores. Os resultados apontaram correlações significativas entre as escalas e os estados de humor; e diferenças significativas entre os níveis de intensidade apontados na escala dos três fatores e os estados de humor. Os resultados indicam clara interferência das dinâmicas sociais sobre os estados de humor, portanto, as interferências sociais não podem ser descartadas na preparação do atleta.

Palavras-chave: interações sociais; humor; psicologia do esporte; voleibol.

\section{Social Dynamics and Mood States}

Abstract: The aim of this study was to evaluate the impact of social dynamics (family, friends and school; i.e., three social factors) on mood states. The survey was conducted with a women's volleyball team (14/15 years). The POMS test (Profile of Mood States) was employed to measure the team's mood states. The athletes assessed the three factors by the use of a Likert scale ranging from 1 (very bad) to 5 (very good). Results showed significant correlations between scales and mood states, and significant differences between the intensity levels representing their moods for each of the three factors. The results show clear interference of social dynamics on the mood states. Therefore, social interference cannot be ignored during an athlete's training program.

Keywords: interpersonal relations; mood; sport psychology; volleyball.

\section{Introdução}

Historicamente, técnicas de controle e monitoramento do treinamento esportivo são fundamentadas sobre variáveis fisiológicas (monitoramento da frequência cardíaca, VO2, lactato, dentre outros) e mecânicas. Focos no monitoramento de aspectos fisiológicos e mecânicos decorrem da possibilidade de um maior controle das variáveis durante os treinamentos e competições. Além disso, a formação acadêmica mostra-se eminentemente "biologizante" em detrimento de uma visão mais ampla e humanística na atividade física e esportiva.

Assim, não podemos deixar de atentar que o esporte é um mesossistema social e, como tal, deve coabitar com outros mesossistemas e com o macrossistema. Numa leitura da sociedade contemporânea Baumann (2007, p.9) nos conduz à seguinte observação: "A "sociedade" é cada vez mais vista e tratada como uma "rede" em vez de uma "estrutura" (para não falar em uma "totalidade sólida"): ela é percebida e encarada como uma matriz de conexões e desconexões aleatórias e de um volume essencialmente infinito de permutações possíveis". Nesse mesmo sentido Berger (2002) afirma que a realidade social apresenta-se como possuidora de muitos níveis de significado. A descoberta de cada novo nível modifica a percepção do todo. Por conseguinte, nos dias atuais, não há como ignorar que as dimensões sociais, emocionais e, inclusive, as espirituais não interfiram no esporte de rendimento. A Figura 1 apresenta algumas dessas relações no esporte.

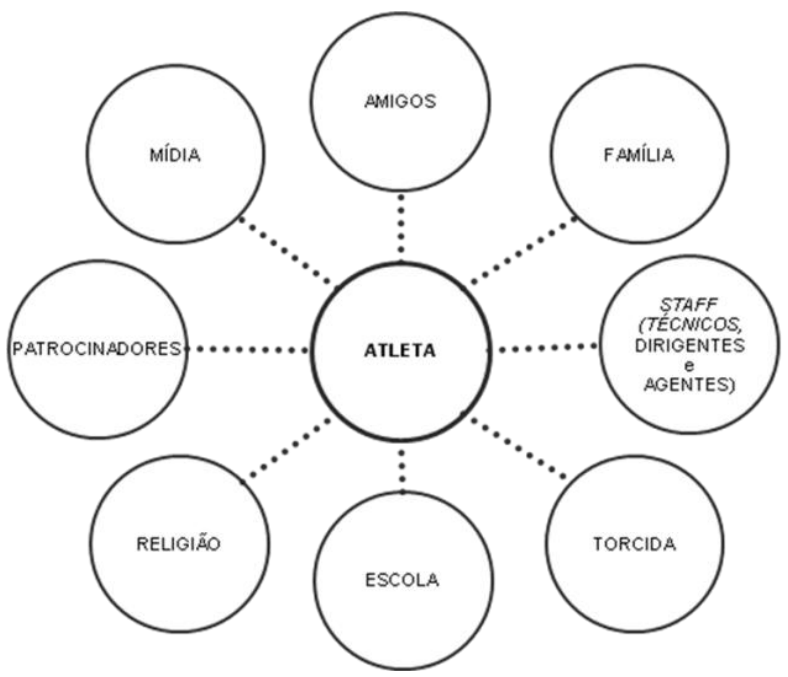

Figura 1. Dinâmicas sociais e o atleta. 
Para Coakley (1991), três fatores refletem extrema importância quando tratamos do ambiente esportivo e os fatores sociais que 0 envolvem:

a) A participação esportiva está positivamente associada a um suporte social vindo de pessoas que são importantes para o atleta;

b) A relativa influência destas pessoas (pai, mãe, amigos, professores, técnicos e pares) e o extenso encorajamento recebido do sistema social;

c) A socialização envolve reciprocidade ou efeito bidirecional no senso de envolvimento entre a criança e o adulto no esporte.

Machado (2006, p. 67) aprofunda essa influência: "a procura vertiginosa por clubes e modalidades em evidência propiciam a saltar o fosso que separa as classes sociais, possibilitando alcançar uma ascensão social, levando primeiramente a uma pretensa conquista financeira, status e fama. Nessa ordem, todos os sacrifícios pelos quais os adolescentes têm que passar para conquistar o reconhecimento da sociedade e, com isso, o sucesso profissional, não têm parâmetros: vale tudo para chegar à fama." Toda a pressão por desempenho e "possibilidade" de ascensão social associada ao que Gallahue e Ozmun (2005) identificam com "uma fase de confrontação com status mutável e com 0 crescente número de papéis a desempenhar," pode desencadear dinâmicas fortes que talvez, não sejam suportadas pelos jovens atletas, resultando no abandono do esporte (REBUSTINI; MACHADO, 2008).

Nos últimos anos, o que se verifica é que atletas cada vez mais jovens são agenciados. Em nome do profissionalismo são representados por procuradores que fazem com que os pais desses atletas assinem procurações que dão a eles (procuradores) plenos poderes em negociações dentro do esporte. Muitas vezes, os próprios pais são os agentes. Esse processo, travestido de profissionalismo, faz com que o jovem atleta e seus familiares vislumbrem a ascensão social por meio do esporte, remediando todas as dificuldades. Na prática o que se verifica é que uma ínfima parcela desses jovens atingirá efetivamente 0 alto rendimento e terá uma ascensão social. Assim, as intrincadas e complexas etapas de desenvolvimento e formação de um atleta tecem novas interações e dinâmicas, em que os componentes "humanos" do atleta não podem ser desconsiderados na progressão da carreira esportiva.

Sedimentado por essas premissas, fica evidente que as dinâmicas sociais influenciam e interferem na formação e nas reações dos jovens. O pressuposto imbricado nesse estudo é a associação dos meios sociais e as alterações emocionais, neste caso, os estados de humor. As dificuldades em estudar e analisar essas associações reside no fato de trabalharmos em dois cenários bastante dinâmicos: o social com toda a sua complexidade e os estados de humor, que notoriamente são estados transitórios, influenciáveis por questões do dia-a-dia. Além dessas duas articulações, Terry (1995), acrescenta que as alterações de humor são, muito provavelmente, dependentes do esporte; portanto, é fundamental que as mudanças de humor em atletas sejam comparadas com seus próprios níveis em diversos momentos. Esta contextualização é imprescindível para compreender como as flutuações intraindividuais dos estados de humor interferem no desempenho atlético, reforça Terry. A dependência a que o autor se refere reside no fato de que cada modalidade esportiva tem suas próprias dinâmicas e culturas, tanto nas inter-relações quanto nos sistemas de treinamento e, desta forma, desencadeiam interpretações, reações e consequências distintas por parte dos atletas. Por conta disto, o monitoramento dos fatores externos é considerado por Berglund e Safstrom (1994) como umas das grandes barreiras para uma melhor acuidade e controle dos estados emocionais e sua inter-relação com as dinâmicas que envolvem o treinamento esportivo e a prática da atividade física.

Em relação aos estados emocionais, um dos instrumentos mais disseminado na psicometria esportiva é o teste de Perfil dos Estados de Humor (POMS - Profile of Mood States), desenvolvido por McNair, Dopplerman e Lorr (1971) e aplicado ao esporte por Morgan (1974).

Inicialmente utilizado para identificar o talento esportivo (MORGAN, 1974, 1980), na década de 90, o teste POMS avançou no estudo de diversos outros fatores: overtraining (RAGLIN; MORGAN, 1994; BERGER; OWEN, 1998), O sistema imunológico (FRY et al., 1994), autoeficácia (REBUSTINI et al., 2007) e questões nutricionais (KENNEDY et al., 2010).

Assim, a tentativa de relacionar as condições do campo social que cercam o jovem atleta 
(família, amigos e escola) e a possível interferência sobre os estados emocionais, parece-nos uma tarefa imprescindível para o futuro equacionamento das etapas de preparação do atleta.

\section{Procedimento Metodológico}

A pesquisa teve como característica a análise quantitativa das informações.

Os participantes $(n=12)$ estudados são atletas de voleibol feminino que competem oficialmente nos campeonatos organizados pela Federação Paulista de Volleyball (FPV) na Categoria Infantil. Das doze atletas, sete tinham 15 anos e cinco, 14 anos. O regime de treinamento era de três vezes por semana, sendo 1 hora e 30 minutos de treinamento de quadra e 1 h00 de preparação física realizada antes do treinamento com bola.

Para a realização da pesquisa foi solicitada autorização ao coordenador das equipes e a técnica; posteriormente, realizou-se uma apresentação dos objetivos da pesquisa para as atletas. Em seguida foi encaminhado aos pais o Termo de Consentimento Livre e Esclarecimento (TCLE), aprovado em projeto submetido ao Comitê de Ética em Pesquisa da Universidade Estadual Paulista "Júlio de Mesquita Filho", do campus de Rio Claro (protocolo n 5375 de 20 de julho de 2011).

O monitoramento contemplou 30 sessões de treinamentos/jogos. O monitoramento foi realizado utilizando-se 0 teste POMS (MCNAIR, DOPPLERMAN \& LORR, 1971), traduzido para o português por Brandão e colaboradores (1993). Como norma de preenchimento do instrumento foi utilizada a instrução "Como você está se sentindo neste momento?". O POMS é mensurado a partir de 65 itens que resultam em seis estados de humor (tensão, depressão, raiva, vigor, fadiga e confusão).

O teste foi preenchido no momento em que a atleta chegava ao ambiente de treinamento e/ou jogo. Foram desenvolvidas três questões e inseridas na própria folha do teste. As questões são: Como está se sentindo em relação à sua convivência em casa com seus pais, irmãos e familiares? Como está o relacionamento com as suas amigas? Como você está na escola com relação as suas notas, tarefas e desempenho?

A percepção do atleta para cada questão foi mensuradas por uma escala do tipo Likert com cinco pontos, sendo 1 - muito mal e 5 - muito bem.

\section{Tratamento Estatístico}

Adotaram-se duas técnicas estatísticas: 1) correlação de Pearson para verificar a relação entre as questões e os estados de humor; 2) análise de variância Anova One Way, Scheffé post hoc test, para avaliar a existência de diferenças significativas entre as respostas das questões relativas às dinâmicas sociais e os níveis de humor. Ambas com nível de significância de $p<0,01$.

Além dos seis fatores que compõem o POMS, foi utilizado o índice denominado Índice de Equilíbrio Emocional Atual (IEEA), calculado a partir do valor da variável vigor, subtraído da somatória das variáveis negativas (tensão, raiva, depressão, confusão e fadiga). O uso do IEEA tem por intuito facilitar a visualização do comportamento global dos estados de humor. Da mesma forma que o desenvolvimento do perfil "iceberg" (MORGAN, 1974), buscamos uma representação otimizada da condição dos estados de humor. A utilização deste índice já foi adotada em estudos preliminares desenvolvidos por

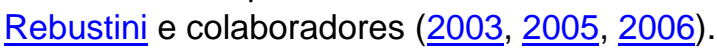

\section{Resultados e discussão}

A análise das correlações estabelecidas entre as dinâmicas sociais e a escala de intensidade foi adotada como primeira via de análise.

Detectaram-se correlações com nível de significância de $(p<0,01)$ entre as três dinâmicas estudadas (Tabela 1).

Tabela 1. Correlação entre as dinâmicas sociais

\begin{tabular}{|c|c|c|c|}
\hline & $\begin{array}{l}\text { Como você está na } \\
\text { escola com relação a } \\
\text { suas notas, tarefa e } \\
\text { desempenho? }\end{array}$ & $\begin{array}{c}\text { Como você está se } \\
\text { sentindo em relação à sua } \\
\text { convivência em casa com } \\
\text { seus pais, irmãos e } \\
\text { familiares? }\end{array}$ & $\begin{array}{l}\text { Como está o } \\
\text { relacionamento com } \\
\text { suas amigas? }\end{array}$ \\
\hline $\begin{array}{c}\text { Como você está na escola com relação a suas } \\
\text { notas, tarefa e desempenho? }\end{array}$ & 1.00 & $0,70^{*}$ & \\
\hline $\begin{array}{l}\text { Como você está se sentindo em relação à sua } \\
\text { convivência em casa com seus pais, irmãos e } \\
\text { familiares? }\end{array}$ & $0,49^{*}$ & 1.00 & \\
\hline Como está o relacionamento com suas amigas? & $0,40^{*}$ & $0,70^{*}$ & 1.00 \\
\hline
\end{tabular}


Um aspecto muito importante é o alto nível de correlação entre o fator "casa" e "amigas". Dentre os aspectos estudados, estes são exatamente os dois que utilizam a via afetiva de relacionamento como primária. Além disso, a família e amigos (afiliação) são considerados fundamentais para o ingresso e permanência da criança e do adolescente no ambiente da atividade esportiva e do esporte (GALLAHUE; OZMUN, 2005; WEINBERG; GOULD, 2001). Em contrapartida, os distúrbios e desequilíbrios nessa relação provocam, entre outros fatores, a diminuição do nível de desempenho, podendo levar até ao abandono da atividade (LOS FAYOS RUIZ; VIVES BENEDICTO, 2002).

Podemos observar que as correlações entre os fatores externos e os estados de humor apresentaram algumas inter-relações que merecem destaque (Tabela 2).

Tabela 2. Correlação entre as dinâmicas sociais e os estados emocionais.

\begin{tabular}{cccccccc}
\hline & TS & DP & RV & VG & FD & CF & IEEA \\
\hline $\begin{array}{c}\text { Como você está na escola com relação a suas notas, } \\
\text { tarefa e desempenho? }\end{array}$ & 0,07 & $-0,32^{*}$ & $-0,06$ & $0,35^{*}$ & 0,01 & $-0,37^{*}$ & $0,34^{*}$ \\
\hline $\begin{array}{c}\text { Como você está se sentindo em relação a sua convivência } \\
\text { em casa com seus pais, irmãos e familiares? }\end{array}$ & $-0,09$ & $-0,35^{*}$ & $-0,18^{*}$ & $0,31^{*}$ & $-0,25^{\star}$ & $-0,32^{*}$ & $0,46^{*}$ \\
\hline Como está o relacionamento com suas amigas? & $-0,12$ & $-0,29^{*}$ & $-0,17^{*}$ & $0,29^{*}$ & $-0,18^{*}$ & $-0,27^{*}$ & $0,41^{*}$ \\
\hline${ }^{*} p<0.01$ & & & & & & &
\end{tabular}

A depressão (DP), o vigor (VG), a confusão (CF) e o IEEA apresentaram correlações significativas $(p<0,01)$ com as três questões. De outra maneira, a fadiga (FD), tensão (TS) e raiva (RV) não apresentaram uma uniformidade com relação aos fatores estudados.

Para depressão e confusão as correlações foram negativas nas escalas de percepção. Assim, quanto melhor o nível de percepção na escala, menor o valor dessas variáveis. A fadiga teve a mesma tendência negativa, o único senão foi a inexistência de significância com 0 fator "Como você está na escola com relação às suas notas, tarefas e desempenho?".

Com uma tendência completamente inversa, as variáveis vigor e IEEA tiveram um coeficiente de correlação positiva. Além dessa relação em conjunto, o vigor também apresentou níveis moderados para "Como você está se sentindo em relação à sua convivência em casa com seus pais, irmãos e familiares?" e "Como está o relacionamento com suas amigas?". Para todos os outros estados de humor as correlações foram fracas ou baixas.

Ainda nos estados de humor negativo, a raiva apresenta coeficientes negativos para todos os fatores. Contudo, não há significância para "escola" e, mesmo, para "casa" e "amigos" em que ocorreu significância, ela foi classificada como fraca.

A tensão apresentou um comportamento distinto de todos os outros estados de humor. Para os fatores "casa" $(r=-0,09)$ e "amigos" ( $r=$ 0,12 ), os coeficientes foram negativos sem nível de significância. Uma melhoria de percepção na escala tende a resultar em níveis de tensão menores. Em outro sentido, a "escola" $(r=0,07)$ tende a acompanhar a escala.

Em alguns estudos a tensão tem surgido com comportamento conflitante em relação às oscilações dos outros estados de humor. Coelho (2003) verificou que as alterações de humor em exercícios de contrarresistência com intensidade de $50 \%$ e $85 \%$ da RM (repetição máxima), a tensão apresentava diferença significativa $(\mathrm{p}<$ $0,05)$ apenas no regime de intensidade de $85 \%$ RM. Goode e Roth (1993) buscaram relacionar os estados de humor e a Escala de Pensamento durante a Corrida (TDRS - Thoughts During Running Scale). Verificou-se que a tensão mostrou uma correlação significativa com o fator relação interpessoal. Novamente, deparamo-nos com uma reatividade e comportamento distinto da tensão.

Os resultados das correlações entre os estados de humor antes do treinamento apresentaram algumas relações instigantes (Tabela 3). A tensão apresenta novamente tendências diferenciadas em relação aos outros fatores. Esta variável não apresenta significância com a fadiga e confusão. Mesmo com a depressão, raiva e vigor, as correlações foram fracas. Com relação ao IEEA, a correlação foi negativa e significativa, mas de todos os estados de humor foi a única a apresentar correlação baixa. Para todos os outros estados de humor, os coeficientes foram significativos em relação ao IEEA, sendo um nível alto para a DP $(r=-0,71)$ e CF $(r=-0,75)$; e níveis moderados para raiva $(r=$ 
$-0,45)$, fadiga $(r=-0,55)$ e $V G(r=0,58)$. Sendo a variável vigor a única a apresentar uma correlação positiva, demonstrando haver uma coerência já esperada entre os fatores e o IEEA, vigor não estabeleceu níveis de correlação significativos com fadiga e depressão.

Tabela 3. Correlação entre os estados de humor.

\begin{tabular}{cccccccc}
\hline & TS & DP & RV & VG & FD & CF & IEEA \\
\hline TS & 1,00 & & & & & & \\
\hline DP & $0,15^{\star}$ & 1,00 & & & & & \\
\hline RV & $0,15^{\star}$ & $0,57^{*}$ & 1,00 & & & & \\
\hline VG & $0,19^{*}$ & $-0,10$ & $0,20^{*}$ & 1,00 & & & \\
\hline FD & 0,14 & $0,32^{*}$ & $0,38^{*}$ & $-0,07$ & 1,00 & & \\
\hline CF & 0,02 & $0,74^{*}$ & $0,38^{*}$ & $-0,36^{*}$ & $0,27^{*}$ & 1,00 & \\
\hline IEEA & $-0,31^{*}$ & $-0,71^{*}$ & $-0,45^{*}$ & $0,58^{*}$ & $-0,55^{*}$ & $-0,75^{*}$ & 1,00 \\
\hline${ }^{*} \mathrm{p}<0,01$ & & & & & & &
\end{tabular}

A depressão, dentre todos os estados de humor, foi a única a ter níveis moderados e altos. Desta forma, a correlação e consonância, isto é, as tendências de oscilação entre as variáveis DP CF e RV interferem de forma muito intensa sobre $o$ índice de IEEA.

Em um estudo realizado por Raglin e Morgan (1994) na tentativa de desenvolver uma escala para monitorar as induções e alterações provocadas pelo treinamento sobre os estados de humor $e$, principalmente, tendo o intuito de prevenir o overtraining; sete itens do POMS correlacionavam-se satisfatoriamente para a identificação do distress em atletas de natação. $O$ aspecto mais importante é que desses sete itens, cinco estão relacionados à depressão e dois à raiva.

Apesar de estarmos lidando com fatores externos (sociais) e não com a interferência direta das cargas de treinamento é importante notar que depressão e raiva apresentam oscilações similares nas duas esferas de análise.

\section{Família}

Durante a adolescência ocorrem conflitos na relação pais e filhos em torno do controle de recursos que o jovem quer ou considera que the são devidos (McKINNEY, 1986). Usualmente, estas relações são agravadas no adolescenteatleta, uma vez que, a dinâmica esportiva faz com que os deslocamentos constantes para os jogos, as viagens, a tentativa de o jovem tomar decisões sem a anuência da família, (em alguns casos, o jovem atleta já recebe ajuda financeira), faz com que a dependência dos recursos materiais, citada por McKinney, seja reduzida, podendo propiciar um número maior de choques na relação pais $e$ filhos, por uma pretensa independência do jovem atleta.

A complexidade desse fator pode ser avaliada pelas palavras de Machado e Higino (2000: $p$. 125-126):

Pais excessivamente autoritários e punitivos facilitam as tendências ao comportamento de inferioridade e expectativa ao fracasso, um parente atleta visto como elemento de comparação pode tanto ser um estímulo positivo como negativo, forçando o atleta a querer elevar sua reputação ao máximo, ou querer um lugar de destaque; namoradas ou noivas também podem ser os dois tipos de influência, podem apoiar o atleta na vitória e na derrota ou sentir-se em segundo plano, vindo a reagir de maneira desestimuladora para a continuidade da prática esportiva; as esposas preocupadas com a segurança, pois a carreira esportiva é muito vulnerável. A família é um forte elemento complicador se não direcionar seus objetivos com clareza e precisão.

Dessa maneira, a interferência familiar no ambiente esportivo do jovem pode gerar conflitos e consequentes alterações emocionais.

Os resultados a essa questão apontaram que apenas a variável tensão e raiva não apresentaram correlação significativa com a escala de intensidade (ver Tabela 4).

Tabela 4. Valores dos estados de humor para o fator "Como você está se sentindo em relação à sua convivência em casa com seus pais, irmãos e familiares?".

\begin{tabular}{|c|c|c|c|c|c|c|c|c|}
\hline & & TS & DP & RV & VG & FD & CF & IEEA \\
\hline \multirow{2}{*}{ Muito Mal } & & 6,00 & 8,25 & 2,50 & 10,00 & 6,50 & 5,75 & $-19,00$ \\
\hline & & 2,83 & $10,14]$ & 2,89 & 5,48 & 4,38 & $3,86-$ & 27.87 \\
\hline \multirow{2}{*}{ Mal } & $x$ & 8.25 & 2,25 & 2,13 & 14,88 & 2,37 & 3,38 & $-3,50$ \\
\hline & $d p$ & 4,27 & 2,714 & 1,13 & 5,77 & 3,81 & 2,77 & 13,80 \\
\hline \multirow{2}{*}{ Normal } & $\mathrm{x}$ & 6,16 & 2,81 & 2,28 & 14,05 & 1,98 & 3,58 & $-2,65$ \\
\hline & $d p$ & 3,58 & $5,48+$ & 4,04 & 5,44 & $2, e 9$ & 3,77 & 15,62 \\
\hline \multirow{2}{*}{ Bem } & $x$ & 6,29 & 0.45 & 1,30 & 14,82 & 2,09 & 2,22 & 2,39 \\
\hline & $d p$ & 2,71 & 1.15 에 & 1,82 & 6,08 & 2,92 तो & 1,51 俩 & 7,27 \\
\hline \multirow{2}{*}{ Muito Bem } & $x$ & 5,81 & 0,33 & 1.15 & 18,42 & 0,94 & 1.78 & 8,35 \\
\hline & $d p$ & 3,35 & 1.29 \# & 1,53 & $4, \pi 7$ & 1,58 & $1,33 \neq$ & 5,99 \\
\hline
\end{tabular}

$p<0,01$ 
Um aspecto importante sobre a intensidade das alterações percebidas é que apenas nessa dinâmica social encontramos a percepção "muito mal (1)".

O aparecimento na escala da citação "muito mal" fez com que surgissem diferenças significativas com os níveis "bem" e "muito bem" da escala, principalmente, para depressão, confusão e fadiga.
A possível migração do que antes fora avaliado como "mal" em outros fatores para uma intensidade maior "muito mal", provocou uma alteração na disposição dos níveis dos estados de humor, não estabelecendo uma visualização tão clara do comportamento das variáveis. Contudo, podemos novamente ressaltar a importância da variável IEEA como um facilitador de visualização do comportamento da oscilação dos estados de humor e a escala de intensidade (Figura 2).

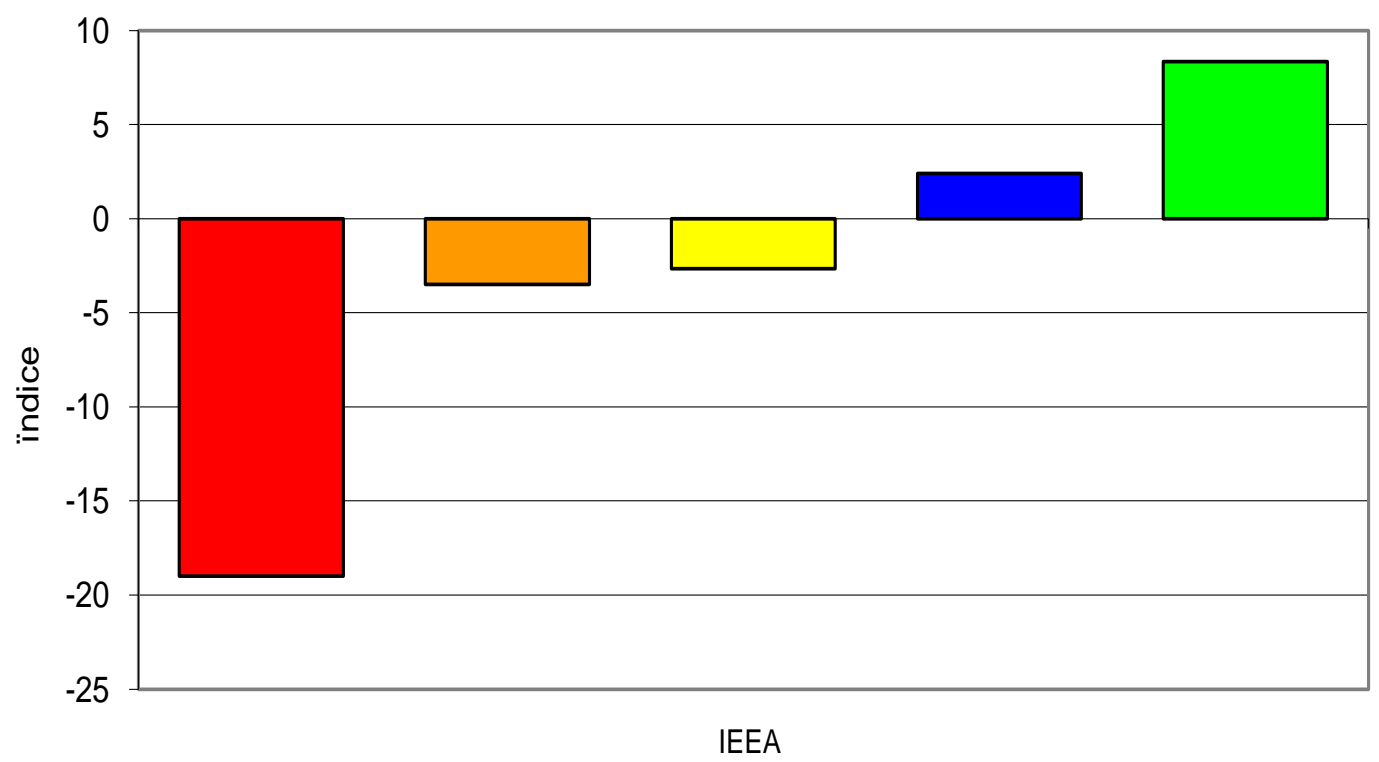

口Muito Mal aMal aNormal $\quad$ Bem aMuito Bem

Figura 2. IEEA para o fator "Como você está se sentindo em relação à sua convivência em casa com seus pais, irmãos e familiares?".

\section{Afiliação}

A literatura tem nos trazido a afiliação e o estabelecimento dos laços de amizade entre as crianças e os jovens como um dos principais fatores para o ingresso e permanência no mundo da atividade física e da competição (WEINBERG; GOULD, 2001).

O processo de afiliação é tão importante no ambiente esportivo e na própria vida social do adolescente que raras são às vezes em que ele (adolescente ou criança) surge sozinho para tentar o ingresso em uma equipe competitiva ou se inscrever nas escolas de esportes.

A afiliação é essencial para a permanência do adolescente no ambiente esportivo. Contudo, o desenvolvimento do universo competitivo, muitas vezes, provoca confronto direto entre os pares na busca de uma posição na equipe, ou seja, são desafiados a competir uns contra os outros. Esse choque pode acarretar uma reação exatamente inversa: o rompimento dos laços afetivos e o estabelecimento de "relações canibalescas" que podem resultar no abandono precoce da criança/adolescente na esfera esportiva.

Baseado nos aspectos citados, da mesma forma que o fator "Como você está se sentindo em relação à sua convivência em casa com seus pais, irmãos e familiares?", as relações de afiliação estão consolidadas por laços afetivos.

Os resultados desse fator apresentaram um número menor de variáveis com diferenças significativas $(p<0,01)$, em relação ao aspecto da família, já discutidos anteriormente (Tabela 5). 
Tabela 5. Valores dos estados de humor para o fator "Como está o relacionamento com suas amigas?".

\begin{tabular}{ccccccccc}
\hline & & TS & DP & RV & VG & FD & CF & IEEA \\
\hline \multirow{2}{*}{ Mal } & $\mathrm{X}$ & 11,50 & 2,50 & 1,50 & 10,50 & 1,50 & 4,00 & $-10,50$ \\
& $\mathrm{dp}$ & 4,95 & 3,54 & 2,12 & 4,95 & 2,12 & 4,24 & 21,92 \\
\hline \multirow{2}{*}{ Normal } & $\mathrm{X}$ & 6,81 & 3,39 & 2,52 & 14,81 & 2,29 & 3,87 & $-3,90$ \\
& $\mathrm{dp}$ & 4,31 & 6,98 & 3,30 & 5,18 & 3,31 & 4,48 & 20,13 \\
\hline \multirow{2}{*}{ Bem } & $\mathrm{X}$ & 6,14 & .92 & 1,42 & 14,39 & 2,11 & 2,40 & 1,32 \\
& $\mathrm{dp}$ & 2,53 & 2,03 & 1,85 & 6,33 & 3,00 & 1,63 & 8,08 \\
\hline \multirow{2}{*}{ Muito Bem } & $\mathrm{X}$ & 5,88 & .25 & 1,15 & 18,20 & 1,11 & 1,84 & 7,92 \\
& $\mathrm{dp}$ & 3,34 & 1,06 & 1,57 & 4,60 & 1,72 & 1,41 & 6,19 \\
\hline
\end{tabular}

$\mathrm{p}<0,01$

Desta maneira, o IEEA surge novamente como um índice de maior clareza de comportamento dos estados de humor ocorrendo diferenças significativas na escala do muito bem (5) para os outros três níveis de escala (bem, normal, mal) apontados na coleta.

Os resultados da depressão e confusão apontam novamente a mesma tendência, sustentada pela alta correlação estabelecida entre as duas variáveis com diferenças significativas da intensidade 3 - normal, para bem (4) e muito bem (5).
Não há um padrão de oscilação entre as variáveis de humor. A variável vigor estabeleceu diferenças significativas do muito bem (5) para bem (4) e para normal (3). A raiva apenas do normal para o muito bem e a fadiga apenas apresentou diferença significativa do muito bem para o bem.

Dos estados de humor que compõem o teste POMS podemos destacar o comportamento do IEEA que estabeleceu uma tendência clara entre a escala de avaliação e os resultados. Isto é, quanto melhor a percepção da relação com os amigos, melhor o IEEA (Figura 3).

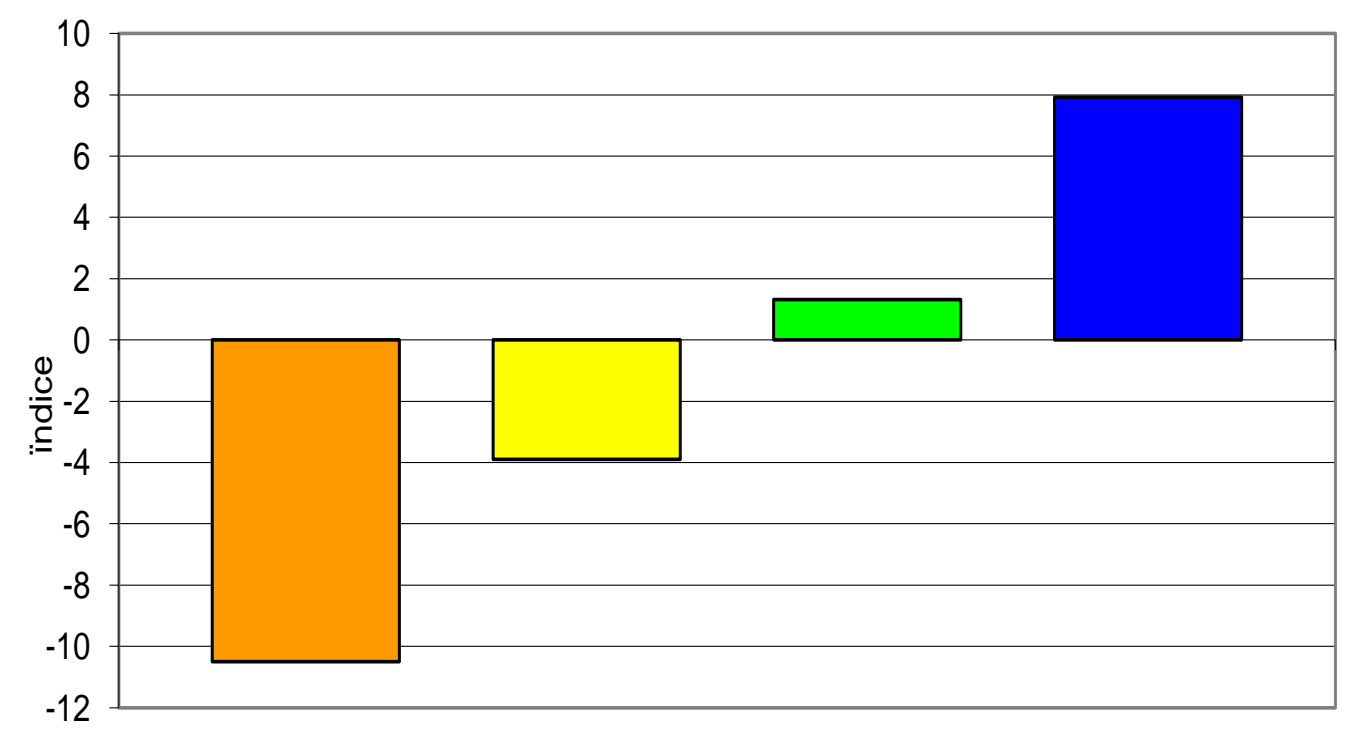

IEEA

口Mal aNormal aBem aMuito Bem

Figura 3. IEEA para o fator "Como está o relacionamento com suas amigas?".

A tensão, apesar de não ter apresentado diferenças significativas entre os níveis de escala, aponta uma tendência clara de redução do seu valor conforme a atleta percebe uma melhoria da 
relação com os amigos. Este comportamento também pode ser notado na confusão. Podemos ressaltar o fato de que a tensão não obteve uma tendência clara para os outros dois fatores analisados.

\section{Escola}

Não podemos esquecer que, no Brasil, o esporte de rendimento está sediado em clubes esportivos, diferentemente dos modelos americanos e europeus, onde o desenvolvimento inicial do esporte se dá na escola e de forma multilateral. Esta formação tem se dado em quase sua totalidade voltada única e exclusivamente ao resultado, sem se importar com 0 desenvolvimento multilateral e longitudinal da criança e do adolescente (REBUSTINI; MACHADO, 2008).

Antes de adentrarmos nos resultados dos estados de humor para esse fator, faz-se necessário um delineamento das dinâmicas que cercam a escola no contexto das atletas de competição nas categorias menores.

Diferente dos jovens de sua idade, os atletas adolescentes têm seu tempo de estudo reduzido em relação aos seus pares. Normalmente, o período da tarde e começo da noite são ocupados pelas sessões de treinamento. Em algumas modalidades, como a natação, há ainda sistemas de treinamento de "madrugada". Os atletas treinam antes de ir para a escola e retornam após as aulas para treinar. As sessões semanais em média são de quatro a cinco, com duração entre duas e três horas. Somemos os tempos de deslocamento para os treinamentos, estamos, portanto, falando de pelo menos cinco horas diárias gastas com o treinamento. Assim, eles têm seu tempo de descanso e lazer reduzidos. Como consequência, realizam suas tarefas escolares em níveis de fadiga mais elevados, podendo acarretar na perda efetiva de aprendizagem.

Os jogos são os eventos que ocupam e exigem um gasto maior de tempo. Geralmente, as partidas são realizadas durante a semana no período noturno e nos finais de semana à tarde ou pela manhã. $O$ resultado disso é que muitas vezes, os atletas chegam a ocupar seis a sete horas do dia para os deslocamentos e a partida, além de comprometerem completamente seus finais de semanas com as competições.
Outro fator que agrava mais ainda essa situação, é que essas atletas contam em sua grande maioria com bolsas de estudos cedidas por escolas particulares que visam antes de tudo, o desempenho elevado nas competições escolares. O reflexo disso é um desgaste ainda maior para as jovens atletas que passam a atender as exigências de um calendário rígido e desgastante de competições oficiais, acrescido de jogos e, muitas vezes, treinamentos agendados pelos seus professores e técnicos escolares.

A inserção dos atletas de competição no ambiente do esporte de competição escolar pode provocar o surgimento de alguns fatores aos quais devemos nos ater:

1. Os atletas, muitas vezes, são promovidos a ícones, e passam a carregar o estandarte da instituição, o que recai novamente sobre eles exercer um papel social distinto dos seus pares. Eles usam imagens de "vencedores" para elevar o nome da instituição que lhes concedeu a bolsa de estudo;

2. Esta dinâmica pode criar um processo discriminatório nas aulas de educação física, pois, os atletas são colocados em lugar de destaque em relação aos seus pares, sendo muitas vezes cultuados em detrimento ao papel de seus pares na equipe. Nesse caso, o professor perde 0 controle do desenvolvimento pedagógico de seus alunos, tornando suas aulas e atividades quase que exclusivamente voltadas à competição.

3. Outro aspecto é a diferença psicofísica dos alunos "regulares" que participam da competição escolar e dos atletas que são inseridos nessas equipes, em virtude do direcionamento da formação. A diferença entre eles é abismal.

Não estamos anulando a importância deles para a dinâmica social e muitas vezes de difusão do esporte no ambiente escolar, e sim, criticamos a excessiva exposição e responsabilidades impostas de forma tão precoce. Dessa forma, o fator: "Como você está na escola em relação às suas notas, tarefas e desempenho?" busca verificar a existência de possíveis alterações provocadas pelo ambiente escolar. Os resultados encontrados na Tabela 6 descrevem os valores de humor em relação à escala de percepção. 
Tabela 6. Valores dos estados de humor para o fator "Como você está na escola com relação as suas notas, tarefas e desempenho?"

\begin{tabular}{|c|c|c|c|c|c|c|c|c|}
\hline & & $\mathrm{TS}^{*+}$ & DP & RV & VG & FD"- & CF & IEEA* \\
\hline \multirow{2}{*}{ Mal } & $x$ & 5,80 & 10,80 & 4,80 & $12, \infty 0$ & 2,80 & 11,00 & $-22,40$ \\
\hline & $d p$ & 1,82 & $10,38]$ & $5,31]$ & 6,99 औ & 4,15 & 6,407 & 24,91 \\
\hline \multirow{2}{*}{ Normal } & $x$ & 5,62 & 1,73 & 1,75 & 13,24 & 1,64 & 2,78 &,- 18 \\
\hline & $d p$ & 3,11 & 4,13 니 & $2,31 *$ & 6,59 내 & 2,78 & $2,16 *$ & 13,19 \\
\hline \multirow{2}{*}{ Bem } & $x$ & 6,24 & .55 & 1,09 & 16,04 & 1,59 & 2,15 & 4,40 \\
\hline & $d p$ & 3,24 & $1,57^{*}$ & $1,81^{\circ}$ & 5,19 & 2,43 & 1,49 & 7,79 \\
\hline \multirow{2}{*}{ Muito Bem } & $\mathrm{x}$ & 6,27 & .24 & 2,09 & 20,31 & 2,00 & 1.49 & 8,00 \\
\hline & $d p$ & 2,97 & $.71=$ & $1,88^{*}$ & 4,79 & 2,84 & $1,31 *$ & 8,11 \\
\hline
\end{tabular}

$p<0,01$ - existe diferença significativa para todas as variações.

${ }^{* *}$ não há diferenças significativas para a TS e FD em nenhum momento.

Novamente, a tensão e fadiga destoam em relação aos outros estados de humor. Ambas não apresentaram diferenças significativas entre os níveis da escala. Este diferencial de comportamento em relação com as outras variáveis foi encontrado nos três fatores estudados.

A variável depressão, raiva e confusão apresentaram diferenças significativas $(p<0,01)$ da escala Mal (2) para todas as outras variáveis da escala. Com exceção do nível 1 (muito mal) que não apresentou citações. Para a depressão ainda ocorrem diferenças significativas da avaliação normal (3) para bem e muito bem. Para raiva ocorre diferença significativa do muito bem (5) para bem (4).
Nas variáveis negativas usualmente tem ocorrido da escala mais baixa apresentar diferenças significativas. No vigor ocorre exatamente o inverso. As diferenças ocorrem do muito bem (5) para os outros graus da escala. No caso do fator "escola" não foi diferente.

Quanto ao IEEA (Figura 4), as diferenças significativas ocorreram entre todas as interações possíveis na escala de intensidades. Além disso, torna-se recorrente a relação clara entre os níveis da escala de percepção e os índices de IEEA.

A importância do monitoramento dos estados emocionais reside no fato de que, nenhum dos fatores descritos anteriormente está associado às questões táticas, técnicas e físicas.

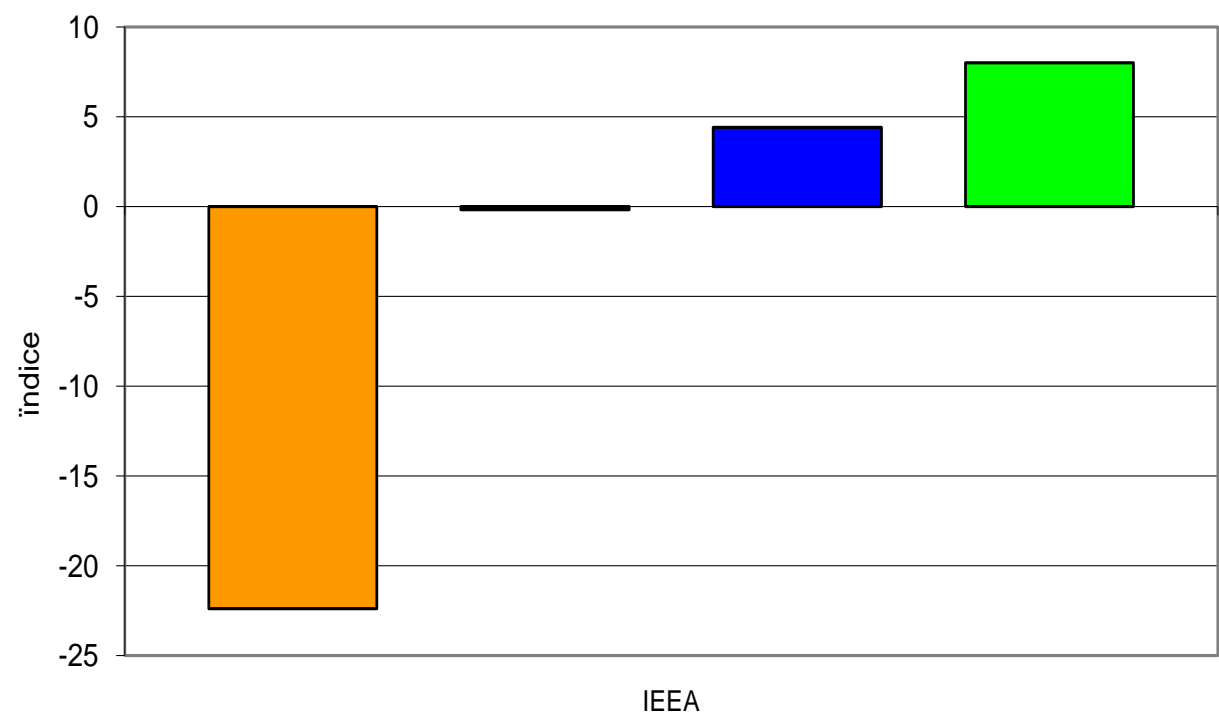

口Mal aNormal aBem aMuito Bem

Figura 4. IEEA para o fator "Como você está na escola com relação a suas notas, tarefa e desempenho?" 
Pelo que vimos, o fator "escola" está em sintonia com os outros fatores, baixas percepções de desempenho resultam níveis negativos de IEEA com níveis mais baixos de $V G$ e elevação dos níveis das variáveis negativas. Isso resulta em médio prazo no abandono do esporte ou da escola.

\section{Considerações finais}

Os dados apresentados e analisados ao longo desse artigo conduzem-nos a alguns apontamentos: 1) Foi possível mensurar as relações entre as dinâmicas sociais sobre os estados emocionais; 2) foram descobertas diferenças significativas entre os níveis de desempenho das participantes nas escalas de avaliação, entre os estados de humor e entre as três dimensões sociais estudados.

Sendo assim, a pesquisa possibilitou algumas considerações: como é recorrente na literatura que os estados emocionais estão intimamente conectados ao resultado esportivo, e aqui fica demonstrado que eles são influenciados pelas dinâmicas sociais que antecedem a prática esportiva. É perigoso para o resultado esportivo não se atentar aos fatores externos no planejamento da preparação desportiva. Assim, a abordagem da ciência esportiva baseada no modelo biológico para explicar as alterações de humor decorrente da prática esportiva é incompleta ( $\underline{\text { SZABO }}, 2003)$.

Essa observação nos conduz a um problema relevante: a pouca atenção dada na formação dos profissionais da educação física quanto às questões psicossociais. A formação eminentemente biologizante limita a atuação dos profissionais, em um ambiente cercado de complexidades. O que acaba limitando de forma substancial a observação, análise e aplicabilidade dos treinamentos, pois uma diversidade de variáveis não são levadas em consideração pelos profissionais que militam no esporte de competição. Não adianta falar de equipes multidisciplinares e muito menos de trabalho interdisciplinar, se o profissional responsável por organizar as equipes, nesse caso, o técnico, não tem um olhar para o cenário macro, está apenas centrado na "aplicação do treinamento".

É incontestável a importância das interações sociais no processo de formação do atleta, seja de qualquer idade, sexo e faixa etária. Assim, não podemos esquecer que no esporte de alto rendimento contamos ainda com a interferência mais intensa da mídia, dinheiro (contratos), empresário (agentes) e patrocinadores.
A visão adotada por dirigentes, técnicos e staff que ignoram esses fatores externos (muitas vezes, chamados de "frescuras"), devem passar por uma grande reflexão. Torna-se um imenso e complexo desafio a aplicação de uma abordagem mais ampla das ciências esportivas.

\section{Referências}

BAUMANN, Z. Tempos líquidos. Rio de Janeiro: Zahar, 2007.

BERGER, B.G.; OWEN, D.R. Relation of low and moderate intensity exercise with acute mood change in college joggers. Perceptual Motor Skills, Missoula, v.87, n. 2, p. 611-621. 1998.

BERGER, P. Perspectivas sociológicas: uma visão humanística. Petrópolis: Vozes, 2002

BERGLUND, B.; SÄFSTRÖM H. Psychological monitoring and modulation of training load of world-class canoeists. Medicine Science in Sports and Exercise; Madison, v.26, n.8, p.10361040, 1994.

BRANDÃO, M. R. F., ANDRADE, D. R., MATARAZZO, F., VASQUEZ, L. F., VASCONCELLOS, E. G. Perfil psicológico dos estados de humor.In: CONGRESSO INTERNO DE PSICOLOGIA DA USP, 2º, 1993, São Paulo. Anais...: Instituto de Psicologia da USP, 1993, p. F1.

COAKLEY, J. Socialization and Sport. In. SINGER, R.N; MURPHERY, M; TENNANT, L.K. Handbook of Research on Sport Psychology, British Columbia, 571-586, 1991

COELHO, E.F. $O$ efeito agudo dos exercícios físicos contra-resistência nos estados de humor. 2003, 127f. Dissertação (Mestrado em Educação Física). Universidade Gama Filho - Rio de Janeiro, 2003.

FRY, R.W, GROVE, J.R, MORTON, A.R; GAUDIERI, S; KEAST, D. Psychological and immunological correlates of acute overtraining. British Journal of Sports Medicine, Leicestershire. Dec, 28(4):241-246, 1994.

GALLAHUE, D.L.; OZMUN,J.C. Compreendendo o desenvolvimento motor: bebês, crianças, adolescentes e adultos. São Paulo: Phorte Editora, 2005. 
GOODE, K.T; ROTH, D.L. Factor analysis of cognitions during running: association with mood change. Journal of Sport \& Exercise

Psychology, Champaign,15, p.375-389, 1993.

KENNEDY, D. O.; VEASEY, R.; WATSON, A.; DODD, F.; JONES, E.; MAGGINI, S.; HASKELL, C. F. Effects of high-dose B vitamin complex with vitamin $\mathrm{C}$ and minerals on subjective mood and performance in healthy males.

Psychopharmacology, Danvers, 211, p. 55-68, 2010.

LOS FAYOS RUIZ, E.J.G.; VIVES BENEDICTO, L. Variables motivacionales y emocionales implicadas en el síndrome de burnoud em el contexto deportivo. Revista Digital - Revista Electrónica de Motivación y Emoción, Castelló de la Plana, vol 5, n. 11-12, dec. 2002. Disponivel em:

$<$ http://reme.uji.es/articulos/agarce2110312102/te xto.html>. Acesso em: 10 fev. 2004.

MACHADO, A. A. Psicologia do Esporte: da educação física escolar ao esporte de alto nível. Rio de Janeiro: Guanabara Koogan, 2006

MACHADO, A.A; HIGINO, W.P.; Violência no esporte e violência social: In. DOBRANZKY, I; MACHADO, A.A. (orgs.). Delineamento da Psicologia do Esporte: evolução e aplicação. Campinas, Tecnograf, p.123-134, 2000

\section{McKINNEY, J.P. Psicologia do}

Desenvolvimento - $\mathrm{O}$ adolescente e o jovem adulto. Rio de Janeiro, Ed. Campus, vol 3, 1986.

McNAIR, D., LORR, M. DOPPLERMAN,L. Profile of Mood States. Educational and Testing Service, San Diego, CA 1971.

MORGAN, W.P. Selected Psychological considerations in sport. Research Quarterly, Washington, 45(4), 374-390, 1974.

MORGAN, W.P. Test of champions. Psychology Today, New York, 92-99, 1980.

RAGLIN, J.S; MORGAN, W.P. Development of a scale for use in monitoring training-induced distress in athletes. International Journal of Sports Medicine, New York, 15, p 84-88, 1994.

REBUSTINI, F. ; SILVA JUNIOR, F. P.; DAVID, P. A.; STORT, R. ; MACHADO, A. A. Training intensity and mood states in young volleyball players. In: INTERNATIONAL CONGRESS OF
APPLIED PSYCHOLOGY, 26th, 2006, Atenas. Proceeding... Athens, 2006. p. 978-978.

REBUSTINI, F., BRANDÃO, M.R.F, AGRESTA, M.C. Emotional fitness profile in female volleyball players as related to performance outcomes In: EUROPEAN CONGRESS OF SPORTS PSYCHOLOGY, XIth, Copenhagen, 2003. Proceeding...Copenhagen, 2003. s/n.

REBUSTINI, F.; MACHADO, A. A. Imediatismo e Performance: incongruências na formação do jovem atleta. In: Machado, A. A. (Org.). Especialização esportiva precoce: perspectivas atuais da psicologia do esporte. 1 ed. Jundiaí: Fontoura, 2008, p. 67-84.

REBUSTINI, F.; SOARES, M. F. A. ; STORT, R. ; LIMA, L. A.; MACHADO, A. A. Análise dos estados de humor e auto-eficácia em jovens voleibolistas. Coleção Pesquisa em Educação Física, Jundiaí, v. 5, p. 97-102, 2007.

REBUSTINI, F; MORENO, R. M; SILVA, R. B; MACHADO, A. A. Estados de humor e percepção de bem-estar: um estudo com jovens mulheres voleibolistas. Lecturas Educación Física y Deportes, Buenos Aires - Argentina, v. 10, n. 86, p. 80-86, 2005. Disponível em: <http://www.efdeportes.com/efd86/voleib.htm>. Acesso em: 20 ago. 2005.

SZABO, A. Acute psychological benefits of exercise performed at self-selected workloads: implications for theory and practice. Journal of Sports Science and Medicine, Bursa, n. 2; 7787, 2003. Disponível em: <http://www.jssm.org>. Acesso em: 16 mar. 2004.

TERRY, P. The efficacy of mood state profiling with elite performers: A review and synthesis. The Sport Psychologist, Champaign ,9, 309-324, 1995.

WEINBERG, R.S., GOULD, D. Fundamentos da Psicologia do Esporte e do Exercício. Rio Grande do Sul: Ed. Artmed, 2001.

Endereço:

Flávio Rebustini

Departamento de Educação Física - IB/UNESP

Avenida 24-A, 1515 Bela Vista

Rio Claro SP Brasil

13506-900

Tel: (19) 3526.4322

e-mail: frebustini@uol.com.br 
Recebido em: 26 de setembro de 2010.

Aceito em: 23 de novembro de 2011.

\section{(c) (7)}

Motriz. Revista de Educação Física. UNESP, Rio Claro,

SP, Brasil - elSSN: 1980-6574 - está licenciada sob

Creative Commons - Atribuição 3.0 